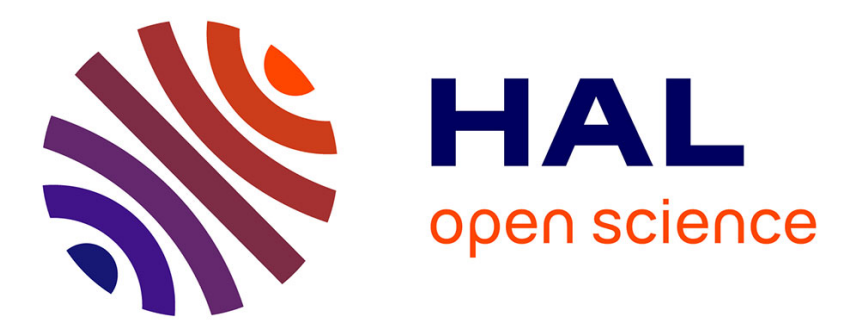

\title{
Decoration of semidilute polymer solutions with surfactant micelles
}

\author{
B. Cabane, R. Duplessix
}

\section{To cite this version:}

B. Cabane, R. Duplessix. Decoration of semidilute polymer solutions with surfactant micelles. Journal de Physique, 1987, 48 (4), pp.651-662. 10.1051/jphys:01987004804065100 jpa-00210482

\section{HAL Id: jpa-00210482 https://hal.science/jpa-00210482}

Submitted on 1 Jan 1987

HAL is a multi-disciplinary open access archive for the deposit and dissemination of scientific research documents, whether they are published or not. The documents may come from teaching and research institutions in France or abroad, or from public or private research centers.
L'archive ouverte pluridisciplinaire HAL, est destinée au dépôt et à la diffusion de documents scientifiques de niveau recherche, publiés ou non, émanant des établissements d'enseignement et de recherche français ou étrangers, des laboratoires publics ou privés. 
Classification

Physics Abstracts

$61.25 \mathrm{H}-82.70 \mathrm{D}-82.70 \mathrm{G}$

\title{
Decoration of semidilute polymer solutions with surfactant micelles $\left({ }^{0}\right)$
}

\author{
B. Cabane and R. Duplessix $\left(^{+}\right)$ \\ CEA, Département de Physicochimie, Laboratoire de Réactivité et Mécanismes en Chimie Inorganique \\ $(*)$, CEN-Saclay, 91191 Gif sur Yvette, France \\ $\left({ }^{+}\right)$Centre de Recherches sur les Macromolécules, 6, rue Boussingault, 67083 Strasbourg, France
}

(Reçu le 26 août 1986, révisé le 8 décembre 1986)

\begin{abstract}
Résumé. - Les solutions aqueuses de polymères hydrosolubles peuvent être décorées par des micelles de tensioactifs qui adsorbent des brins de polymères. Le processus élémentaire enroule une section d'une macromolécule autour d'une micelle ; si la macromolécule est assez longue elle habille consécutivement plusieurs micelles qu'elle relie par des brins libres. Ici nous considérons des solutions semi-diluées de polymères, où des macromolécules de polyoxyéthylène forment un tissu irrégulier à travers toute la solution. On ajoute des micelles de dodécysulfate de sodium à cette solution ; par leurs répulsions électrostatiques ces micelles tendent à former un arrangement régulier qui est couplé par les forces d'adsorption au tissu polymérique. Nous présentons une étude des structures de ces solutions par diffusion aux petits angles de neutrons. Suivant la composition de la solution, nous trouvons 3 types de corrélations spatiales : soit le tissu polymérique s'adapte à l'organisation originelle des micelles et suit leurs espacements ; soit au contraire il désorganise l'arrangement des micelles et impose des corrélations semblables à celles des polyélectrolytes linéaires; soit enfin il recueille une fraction seulement des micelles et rejette autour de lui les micelles en excès.
\end{abstract}

\begin{abstract}
Aqueous polymer solutions can be decorated with surfactant micelles which bind to the polymer strands. The elementary process wraps a section of a macromolecule around a micelle ; if the macromolecule is long enough, it clothes many micelles and connects them by free strands. Here we consider semidilute polymer solutions, where macromolecules of poly (ethylene oxide) form an irregular web throughout the sample. Sodium dodecyl sulfate micelles are added to the solution; because of their electrostatic repulsions they tend to form a 3-dimensional array which is coupled to the web through the binding. The structures of such decorated webs are studied through small angle neutron scattering; depending on the composition of the solution, 3 patterns of correlations are found : adaptation of the web to the original intermicellar spacings, disruption of the intermicellar spacings by the web, spillover of excess micelles by saturated necklaces.
\end{abstract}

\section{Introduction.}

In water many types of dissolved macromolecules will bind to the surfaces of ionic surfactant micelles [1]. The elementary step of the binding wraps 'a section of a macromolecule around a micelle $[2,3]$; if the macromolecule is long enough it can clothe many micelles connected by free strands (Fig. 1), resulting in a «necklace » which may contain up to 300 micelles [4]. Here we consider semidilute polymer solutions, where the macromolecules form

$\left(^{0}\right)$ This work used the neutron beams of ILL in Grenoble and LLB in Saclay.

(*) Associé au CNRS, UA 331. an irregular web throughout the sample. Surfactant micelles are added to these solutions; because of their electrostatic repulsions, they tend to form a liquid-like array which is coupled to the web through the adsorption.

This coupling has consequences for the structures of the solutions ; the obvious questions are : will the irregular web disrupt the array of micelles ? Or will the array impose its spacings to the web ? Moreover, the rheology of the solutions depends on a related topological problem : is it possible for a micelle to bind many macromolecules, resulting in a reticulation of the web by the micelles?

Here we present neutron scattering measurements of the correlation function for the distances between 


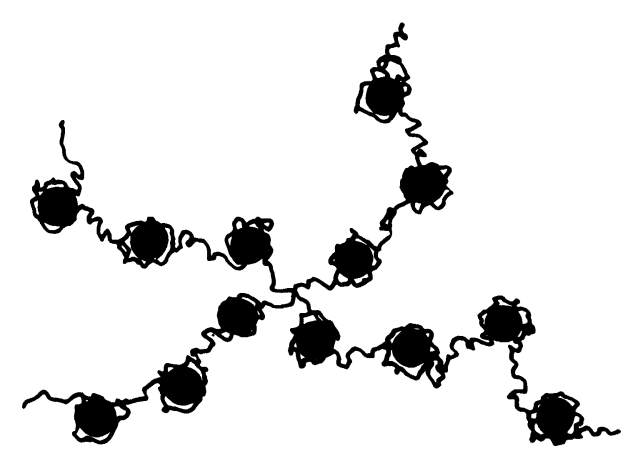

Fig. 1. - PEO macromolecules bound to the surfaces of SDS micelles. Each adsorbed section contains approximately 60 monomers ; the distances between consecutive micelles must be at least $60 \AA$ when the repulsions between micelles are screened (50 monomers per free strand), and $90 \AA$ when they are not (150 monomers per free strand) [4].

micelles and of that between monomers of the macromolecules in a semidilute polymer + surfactant solution. Our results show some of the behaviours listed above, with sharp crossovers according to the concentrations $\mathbf{x}$ for the surfactant and $\mathbf{y}$ for the polymer.

1.1 Micelles. - First consider the micelles. They are formed by the spontaneous aggregation of surfactant molecules dissolved in water ; we use molecules of sodium dodecyl sulfate (SDS) which form globular micelles containing about 70 SDS molecules each [510]. The adsorption of the polymer strands does not disrupt their structure, provided that the free energy of adsorption is small compared to the cohesive free energy of the micelle ; in this respect the situations to avoid are the vicinity of the critical micellar concentration (c.m.c.) of the surfactant [11] (then the free energy gain associated with micelle formation is small) and the binding to polymers of opposite charge [12] (then the free energy of binding is large). For SDS micelles bound to poly (ethylene oxide) macromolecules (PEO), the free energy of binding is on the order of $0.3 \mathrm{kT}$ per SDS molecule, much smaller than the free energy of micelle formation which is about $10 \mathrm{kT}$ per SDS molecule [13]. Consequently the binding leaves the structures of the micelles essentially unchanged, and only affects their relative positions.

In homogeneous solvents the locations of the micelles are determined only by their mutual repulsions, and by thermal agitation. The resulting correlations are expressed by a pair correlation function $g_{\mathrm{mm}}(r)$, or by its Fourier transform, the structure factor $S_{\mathrm{mm}}(Q)$ :

$$
S_{\mathrm{mm}}(Q)=\left(n_{\mathrm{m}} V\right)^{-1} \sum_{i \in \mathrm{m}} \sum_{j \in \mathrm{m}} \mathrm{e}^{i \mathbf{Q} \cdot \mathrm{r}_{i j}}
$$

where the index $\mathrm{m}$ indicates that the objects are micelles, $n_{\mathrm{m}}$ is their number concentration in a solution of volume $V, \mathbf{r}_{i j}$ a vector joining the centres of 2 micelles and $\mathbf{Q}$ a vector of reciprocal space $[14,32]$.

At low $Q$ this structure factor is depressed by the electrostatic repulsions, which suppress the long range fluctuations of the density of micelles; then there is a peak which reflects the correlations between neighbouring micelles ; finally, at large $Q$, the phase factors in (1) become out of phase because of the disorder in the positions of the micelles, and therefore $S(Q)$ tends to one.

In a scattering experiment, the information on intermicellar correlations and that on intramicellar structure are mixed $[14,32]$. The formulation is simple when the micelles can be assumed to be spherical and monodisperse; then the intensity scattered from the solution can be written as a product of the single micelle intensity $\mathrm{I}_{\mathrm{m}}(Q)$ by the intermicellar structure factor $S_{\mathrm{mm}}(Q)$ :

$$
\mathbf{I}(Q) / V=n_{\mathrm{m}} \mathbf{I}_{\mathrm{m}}(Q) S_{\mathrm{mm}}(Q) .
$$

Figure 2 shows the results of a neutron scattering experiment on SDS micelles; they are analyzed according to equation (2) by postulating a general model for micellar structure and intermicellar forces $(8,9)$, calculating $S(Q)$ through statistical mechanics [15-17] and comparing the product $\mathrm{I}_{\mathrm{m}}(Q) S_{\mathrm{mm}}(Q)$ with the measured intensities. From the fit of the model to the data the values of the micellar aggregation number $N$ and of the micellar charge $Z$ are determined: we find $N=74$ and $Z=24$ for dilute solutions $\left(x=0.02 \mathrm{~g} / \mathrm{cm}^{3}\right)$, hence $n_{\mathrm{m}}=3 \times 10^{19} \mathrm{x}$.

Note that the intermicellar peak is a liquid structure peak rather than a Bragg peak : they array of micelles is far from being perfectly ordered, and indeed the corresponding peak in $g_{\mathrm{mm}}(r)$ is rather small (8). Still, because the forces are long range,

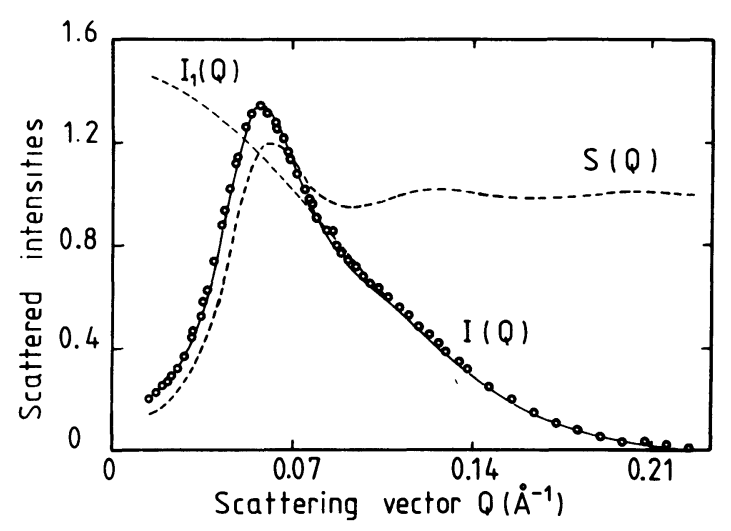

Fig. 2. - Intensity scattered by a solution of a SDS $\left(0.02 \mathrm{~g} / \mathrm{cm}^{3}\right)$ in $\mathrm{D}_{2} \mathrm{O}$. The dotted lines are the calculated curves for the intramicellar structure $\mathrm{I}_{\mathrm{m}}(Q)$ and for the intermicellar interferences $S_{\mathrm{mm}}(Q)$; the full line is the fit of the data by the product $\mathrm{I}_{\mathrm{m}}(Q) S_{\mathrm{mm}}(Q)$. From [10]. 
the peak positions do vary with concentration according to the law of an ordered array

$$
Q_{\mathrm{m}}=2 \pi n_{\mathrm{m}}^{1 / 3}=2 \times 10^{7} \mathbf{x}^{1 / 3}
$$

with

$$
Q_{\mathrm{m}} \text { in } \mathrm{cm}^{-1} \text { and } \mathbf{x} \text { in } \mathrm{g} / \mathrm{cm}^{3} .
$$

Does this « liquid structure " analysis remain valid for micelles bound to a polymer web ? A positive answer would require that the macromolecules produce identical central forces between all neighboring micelles. However this may apply only to solutions where neighbouring micelles are always directly connected by a polymer strand; thus the question really hinges on the topology of the resulting network. To make the effects most visible, we work in solutions of low ionic strength (no added salt), where the correlations of the unperturbed micellar liquid are well marked ; this choice departs from our previous work, where salt was added to screen the correlations between macromolecules loaded with micelles.

1.2 Polymers. - Now we make the solvent heterogeneous by dissolving long macromolecules in it. Each macromolecule spreads through the solvent as a random walk ; if there are sufficiently many of them, they overlap and form an irregular web throughout the solution (this is the definition of semidilute solutions, as opposed to the dilute solutions studied previously [2-5]). For our purposes this web is characterized by its monomer concentration $n_{\mathrm{p}}$, and its mesh (or blob) size $\xi_{\mathrm{b}}$ [18] ; in a good solvent $\xi_{\mathrm{b}} \sim n_{\mathrm{p}}^{-3 / 4}$. At short distances the correlations within one ideal macromolecule decrease like $1 / r$, giving a $Q^{-2}$ decay of the structure factor at large $Q$. At distances much larger than $\xi_{b}$, the correlations between segments of a given macromolecule are screened by the strands of the others [19] ; consequently the pair correlation function of all monomers is damped by a factor $\mathrm{e}^{-r / \xi}$, where the screening length $\xi$ is equal to $0.35 \xi_{\mathrm{b}}$ [20]. The structure factor of the whole web has the form:

$$
S_{\mathrm{pp}}(Q)=\frac{n_{\mathrm{p}} \xi}{Q^{2}+\xi^{-2}}
$$

Figure 3 shows the intensities scattered by semidilute PEO solutions, plotted according to equation (4). The low $Q$ limit indicates that the mesh size $\xi_{\mathrm{b}}$ is $164 \AA$ at $\mathbf{y}=0.01 \mathrm{~g} / \mathrm{cm}^{3}$. At higher $Q$ values the exponent of $Q$ is found to be slightly lower (1.70) than predicted by equation (4) because the monomers of a macromolecule tend to avoid each other [3].

In figure 4 the scattering curves for a pure SDS solution and for a semidilute solution of PEO are compared. Two features are worth noting. First, the

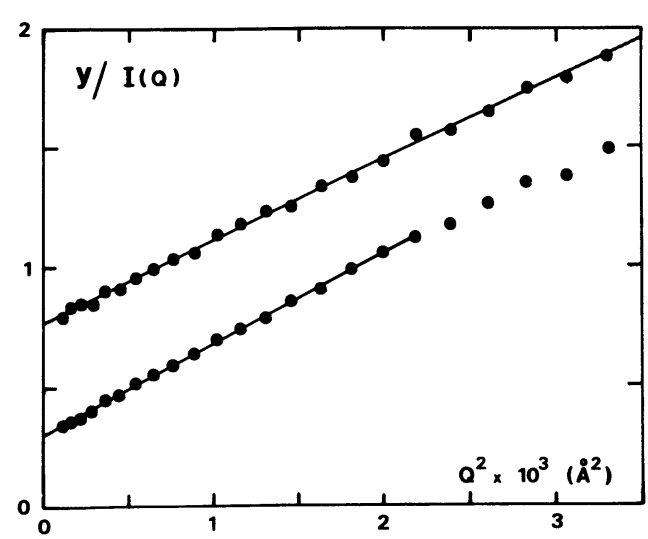

Fig. 3. - Reciprocal intensities from semidilute solutions of PEO in $\mathrm{D}_{2} \mathrm{O}$. In this representation the mesh sizes $\xi_{\mathrm{b}}$ of the solutions can be calculated from the slopes and intercepts of the plots. Upper line : PEO concentration $y=0.04 \mathrm{~g} / \mathrm{cm}^{3}, \xi_{\mathrm{b}}=60 \AA$, Lower line : $\mathbf{y}=0.02 \mathrm{~g} / \mathrm{cm}^{3}$, $\xi_{\mathrm{b}}=100 \AA$.

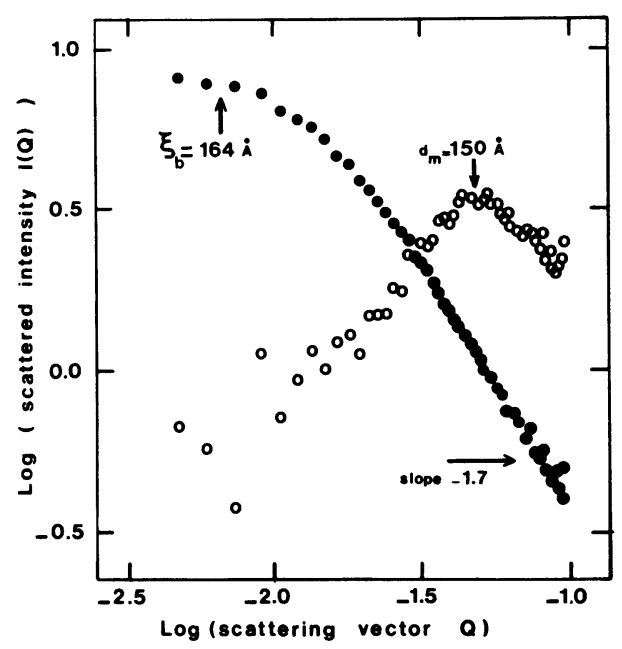

Fig. 4. - Comparison of the scattering curves for the pure components, in a Log/Log plot. Dots : scattering by a semidilute solution of PEO, concentration $y=0.01 \mathrm{~g} / \mathrm{cm}^{3}$ or $n_{\mathrm{p}}=1.4 \times 10^{20}$ monomers $/ \mathrm{cm}^{3}$, mesh size $\xi_{\mathrm{b}}=164 \AA$. Circles : scattering by a solution of SDS, concentration $\mathbf{x}=0.01 \mathrm{~g} / \mathrm{cm}^{3}$ or $n_{\mathrm{m}}=6 \times 10^{17}$ micelles $/ \mathrm{cm}^{3}$, average intermicellar spacing $150 \AA$, micellar radius $20 \AA$.

osmotic fluctuations of the polymer at low $Q$ are quite large, despite the screening caused by the interpenetration of the macromolecules, whereas the micellar solution is largely incompressible at large distances or low $Q$. Second, the range of the correlations within the polymer web $\left(\xi_{\mathrm{b}}=164 \AA\right.$ at $\left.\mathbf{y}=0.01 \mathrm{~g} / \mathrm{cm}^{3}\right)$ is comparable with the average spacing of the micellar array $\left(d_{\mathrm{m}} \approx 150 \AA\right.$ at $\mathbf{x}=$ $\left.0.01 \mathrm{~g} / \mathrm{cm}^{3}\right)$.

1.3 Weak Coupling. - The classical method for describing the coupling between 2 weakly interacting systems is linear response theory. In the present case 
it assumes that each micelle is coupled to every polymer strand in its vicinity, and vice versa [21]

$$
\begin{aligned}
& \boldsymbol{\delta} \boldsymbol{\rho}_{\mathrm{m}}(Q)=S_{\mathrm{mm}}^{0}(Q)\left[\boldsymbol{\delta} \boldsymbol{\phi}+u \boldsymbol{\delta} \boldsymbol{\rho}_{\mathrm{p}}(Q)\right] \\
& \boldsymbol{\delta} \boldsymbol{\rho}_{\mathrm{p}}(Q)=S_{\mathrm{pp}}^{0}(Q)\left[u \boldsymbol{\delta} \boldsymbol{\rho}_{\mathrm{m}}(Q)\right]
\end{aligned}
$$

where the $\boldsymbol{\delta} \rho$ describe the variations in the concentrations of micelles and polymer in response to an external potential $\boldsymbol{\delta} \phi$ acting on the micelles, and $u$ is the strength of the polymer-micelle interaction. This results in an indirect coupling between micelles through polymer strands, and between polymer segments through the micellar array.

In a scattering experiment, contrast matching allows the scattering from the polymer to be observed separately from that of the micelles. Then, according to linear response theory, the intermicellar correlations will still show up in the scattering of the polymer, while the long range fluctuations of the concentration of polymer segments will raise the osmotic compressibility of the micellar array at low scattering vectors :

$$
\begin{gathered}
\left\langle\boldsymbol{\delta} \boldsymbol{\rho}_{\mathrm{m}} \cdot \boldsymbol{\delta} \boldsymbol{\rho}_{\mathrm{m}}\right\rangle=S_{\mathrm{mm}}=\frac{S_{\mathrm{mm}}^{0}}{1-u^{2} S_{\mathrm{mm}}^{0} S_{\mathrm{pp}}^{0}} \\
\left\langle\boldsymbol{\delta} \boldsymbol{\rho}_{\mathrm{p}} \cdot \boldsymbol{\delta} \boldsymbol{\rho}_{\mathrm{p}}\right\rangle=S_{\mathrm{pp}}=\frac{S_{\mathrm{pp}}^{0}}{1-u^{2} S_{\mathrm{mm}}^{0} S_{\mathrm{pp}}^{0}} .
\end{gathered}
$$

The effects of this coupling are small, excepted in those ranges of $Q$ where the denominator comes close to zero. This may occur near the peak of intermicellar correlations, resulting in the growth of a related peak in the scattering curve of the polymer (Fig. 5) and in a rise of the absolute magnitude of

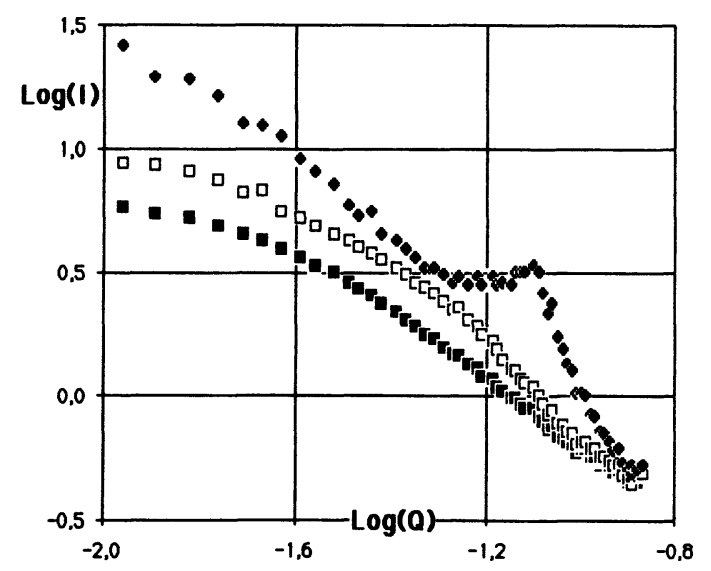

Fig. 5. - Prediction for the scattering curves of polymer solutions coupled to a micellar array. Filled squares : polymer alone. Hollow squares : polymer coupled to a dilute micellar solution where the intermicellar correlations are weak. Filled lozanges : polymer coupled to a concentrated micellar solution where the intermicellar correlations are strong; here the intermicellar peak shows up in the polymer curve as well.

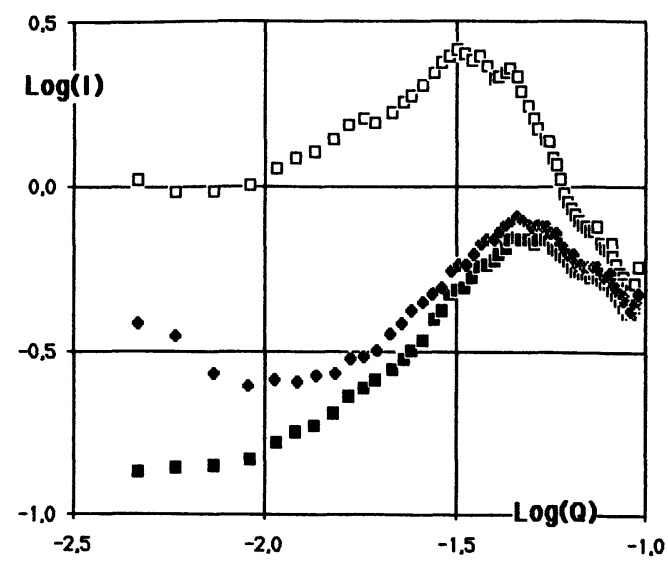

Fig. 6. - Prediction for the scattering curves of micellar solutions coupled to a polymer web. Filled squares : micelles alone. Filled lozanges : micelles coupled to a dilute polymer solution, where the fluctuations of the monomer concentration are large at low $Q$ and small at high $Q$. Hollow squares : micelles coupled to a more concentrated polymer solution, where the fluctuations of the monomer concentration are large at all $Q$ values.

the intermicellar peak (Fig. 6). It may also occur at low $Q$, where the long wavelength fluctuations of the polymer concentration are large (Fig. 4), and will raise the structure factor of the micelles despite their electrostatic repulsions (Fig. 6).

However these large effects are obtained only when the product $u^{2} S_{\mathrm{mm}}^{0} S_{\mathrm{pp}}^{0}$ is so close to 1 that the predicted structure factors are very unstable. In such conditions the coupling is actually strong, and the linear response theory may not apply. Consequently if the effects of the coupling between the web and the array of micelles are significant, they may well differ from the predictions of the linear theory.

\section{Materials and methods.}

2.1 Physical ChEMISTRY. - The situation of identical, repelling spheres coupled to an irregular web can be approximated by the SDS + PEO system within certain constraints for the purity of the materials, their dissolution in water and their ranges of concentration.

The first constraint applies to the purity of SDS. This amphiphile is made through the sulfonation of dodecanol [22] ; if some of this alcohol is left at the end of the reaction, it will not be eliminated by classical purification procedures [23-25]. In dilute SDS solutions, small amounts (a few \%) of dodecanol induce the formation of very large aggregates such as disks [26] or macroscopic pieces of bilayers, because there are not enough micelles to solubilize all the dodecanol. Such solutions are recognized by the well known dip in the surface tension plot, because these large aggregates are more surface active than SDS micelles; they also 
scatter much more in the low $Q$ limit, because the large aggregates dominate this scattering. Similar effects are produced by other impurities which behave as weak amphiphiles.

Our original source of 'SDS was the commercial material available from $\mathrm{BDH}$; this has very little long chain alcohols in it, as indicated by the surface tension plot [2]. Neutron scattering experiments also require the use of perdeuterated SDS ; this was bought mostly from Service des Molécules Marquées at CEN-Saclay; we checked the surface tension plots and the low $Q$ scattering of this material, and found that the batches produced until 1982 and after 1986 met our criteria ; however the material produced between these dates was contaminated by an hydrophobic impurity. Some experiments were done with the perdeuterated SDS from MSD, which appeared to be reliable in this respect.

With the polymer (PEO), the constraints are of a different nature. Usual grades of high molecular weight PEO contain supramolecular aggregates which may take weeks to dissolve, or may never dissolve until the polymer is degraded [27-29] ; these lumps dominate the scattering at low $Q$; the remaining material, wich does dissolve well in water, still has a broad distribution of molecular weights. We use a PEO which has been fractionated by chromatography in water (Toyo Soda SE150); the average molecular weight given by the manufacturer is $1.2 \times 10^{6}$, with a dispersity $M_{\mathrm{w}} / M_{\mathrm{n}}=1.12$. We dissolve this material by stirring it slowly in water for 24 hours at $30^{\circ} \mathrm{C}$; light scattering measurements on such solutions [30] confirm that they do not contain any aggregates, and yield a molecular weight which is close to the nominal one. In scattering experiments the solutions were kept either at $20^{\circ} \mathrm{C}$ or at $50{ }^{\circ} \mathrm{C}$; the results obtained at both temperatures are identical. The solutions can be kept at $50^{\circ} \mathrm{C}$ for a few days or at $20^{\circ} \mathrm{C}$ for a few weeks without any sign of degradation.

2.2 CONTRASTs. - The contrast matching method is used to observe separately the scattering from the micelles and that from the polymer in the solution. This method is applicable when the 2 components have very different densities of scattering length for the radiation used in the experiment $[31,32]$. Because neutrons are scattered by the nuclei in the sample, isotopic substitution can be used to obtain these differences in scattering lengths; here we use deuterated SDS and regular (protonated) PEO dissolved in mixtures of $\mathrm{H}_{2} \mathrm{O}$ and $\mathrm{D}_{2} \mathrm{O}$; the corresponding scattering densities are shown in table $\mathrm{I}$.

The important numbers in this table are the scattering densities $\boldsymbol{\rho}$; indeed the intensities scattered by each component are controlled by its excess scattering density with respect to the solvent $\boldsymbol{\rho}-\boldsymbol{\rho}_{\mathrm{s}}$. In order to observe separately the scattering by the
Table I. - Scattering densities.

\begin{tabular}{lccc}
\multicolumn{1}{c}{ Molecule } & $b$ & $v$ & \multicolumn{1}{c}{} \\
\multicolumn{1}{c}{-} & - & - & $\frac{p}{-387}$ \\
$\mathrm{C}_{12} \mathrm{H}_{25} \mathrm{SO}_{4} \mathrm{Na}$ & 1.595 & $248 \pm 2$ & 0.387 \\
$\mathrm{C}_{12} \mathrm{D}_{25} \mathrm{SO}_{4} \mathrm{Na}$ & 27.62 & $248 \pm 2$ & 6.704 \\
$\mathrm{CH}_{2} \mathrm{CH}_{2} \mathrm{O}$ & 0.414 & 36.7 & 0.68 \\
$\mathrm{H}_{2} \mathrm{O}$ & -0.168 & 18.1 & -0.56 \\
$\mathrm{D}_{2} \mathrm{O}$ & 1.92 & 18.1 & 6.39
\end{tabular}

$b=$ coherent scattering length per molecule in $\mathrm{cm}$ $\times 10^{-12}$, obtained by summing the scattering lengths of nuclei as listed in [33].

$v=$ molar volume, in cc/mole, at $24^{\circ} \mathrm{C}$, from [34-40].

$\rho=(b / v) \times N_{\text {Avogadro }}=$ density of scattering length in $\mathrm{cm}^{-2} \times 10^{10}$.

micelles one must choose a solvent whose scattering density $\boldsymbol{\rho}_{\mathrm{s}}$ matches that of PEO ; this is achieved in the mixture $82 \% \mathrm{H}_{2} \mathrm{O}+18 \% \mathrm{D}_{2} \mathrm{O}$. Conversely the scattering length density of $\mathrm{SDS}_{\mathrm{d}}$ is almost matched by $\mathrm{D}_{2} \mathrm{O}$ : in this solvent the value of $\rho-\rho_{\mathrm{s}}$ is 5.71 for PEO and only 0.31 for $\mathrm{SDS}_{\mathrm{d}}$; then the scattering is controlled by PEO.

2.3 EXPERIMENTS. - The useful range of distances for studying the structures of semidilute SDS + PEO solutions extends from $60 \AA$ (below the shortest intermicellar spacings) to $600 \AA$ (beyond the largest mesh size available with the polymer used in these experiments). Accordingly, scattering vectors should be collected in the range $5 \times 10^{-3}<Q<$ $0.2 \AA^{-1}$. We covered this range with the instruments D17 at ILL and PACE at LLB. In these experiments, the critical factors were the accuracy of the $Q$ scale (minimize the experimental broadenings to avoid smearing out the peaks) and the calibration of the intensity scale (in order to compare different sets of data.

The main sources of broadening in the $Q$ scale are the dispersion in the wavelengths of incident neutrons and the collimation. The first set of experiments on D17 was carried out with a wavelength spread of $10 \%$ and a divergence of $10^{-2}$ radian in the incident beam $\left(\Delta Q \approx 5 \times 10^{-3} \AA^{-1}\right)$; at the peak of the SDS scattering curves $(Q=5 \times$ $10^{-2} \AA^{-1}$ ) the divergence and the wavelength spread contributed equally to $\Delta Q / Q$. Subsequent experiments were made with a $5 \%$ wavelength selector and a smaller divergence $\left(\Delta Q \approx 3 \times 10^{-3}\right)$. On PACE we used different collimations for low $Q$ values $\left(5 \times 10^{-3}<Q<5 \times 10^{-2}\right)$ and for high ones $\left(2 \times 10^{-2}<Q<2 \times 10^{-1}\right)$; then the spread of $Q$ values due to the divergence was respectively $\Delta Q \approx 5 \times 10^{-3}$ and $2 \times 10^{-2} \AA^{-1}$.

The calibration of the intensity scale entailed 3 operations : correction for non uniformity of the detector's response, by comparison with the scattering of $\mathrm{H}_{2} \mathrm{O}$; comparison of relative intensities 
obtained with different configuration of the instrument, which was done by comparing them with the intensity of the attenuated primary beam ; putting the measured intensities on an absolute scale, which was done either by calculating the absolute intensities scattered by $\mathrm{H}_{2} \mathrm{O}[31,41]$ or by using calibrated attenuators.

\section{Scattering curves for semidilute solutions.}

Our experiments do show a clear coupling between the polymer web and the array of micelles; however it is not as predicted by linear response theory. In fact, depending on the composition of the solution, we find 3 different types of coupling (Fig. 7). Each of these behaviours can be given a simple interpretation if the system is a solution of independent necklaces, where a macromolecule which meets a micelle will saturate its surface, and then go on with a free strand before binding to another micelle [45, 46].

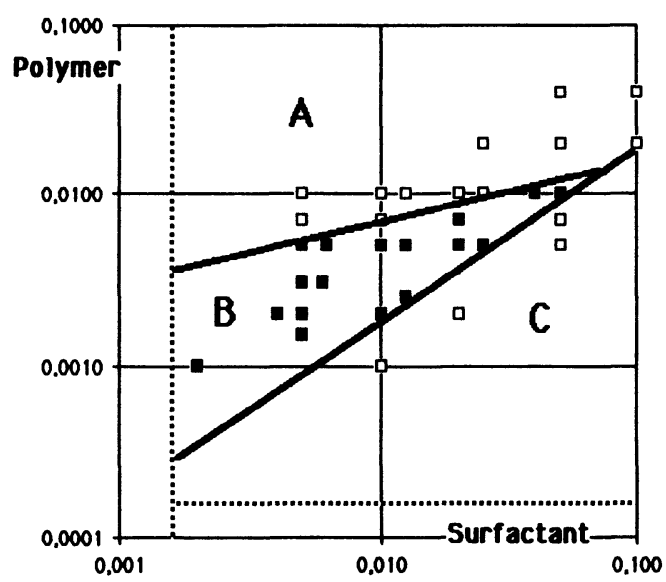

Fig. 7. - Map of the compositions of the semidilute PEO + SDS solutions. The full lines mark the crossovers between regions A (open symbols), B (filled symbols) and $\mathrm{C}$ (open symbols). The vertical dashed line is the c.m.c. of SDS in the presence of PEO, and the horizontal one is the overlap concentration for pure PEO $\left(M=10^{6}\right)$ solutions (the overlap concentration for PEO macromolecules saturated and stretched by SDS micelles is substantially lower).

3.1 Region A. - At high polymer/micelle ratios, the micellar array is not perturbed by the polymer: its scattering curve is similar to that of free SDS micelles, with a correlation hole at low $Q$ and a single peak near the same correlation distance $d_{\mathrm{m}}=150 \AA$ for $\mathbf{x}=0.01 \mathrm{~g} / \mathrm{cm}^{3}$ (Fig. 8). If anything, the arrangement of the micelles is more regular than in a free array, because the long wavelength (low $Q$ ) fluctuations of their concentration appear to be nearly completely suppressed.

The coupling shows up in the correlations of the polymer web, whose Fourier components are strongly depressed in the range $5 \times 10^{-3}<Q<$ $2 \times 10^{-2} \AA^{-1}$ (Fig. 9). Obviously, in this range of $Q$ vectors, the fluctuations of the concentration of macromolecules are suppressed by the electrostatic repulsions between the micelles to which they are bound. However it is remarkable that the low $Q$ limit of this scattering rises again to reach that of the free polymer : apparently the very long range fluctu-

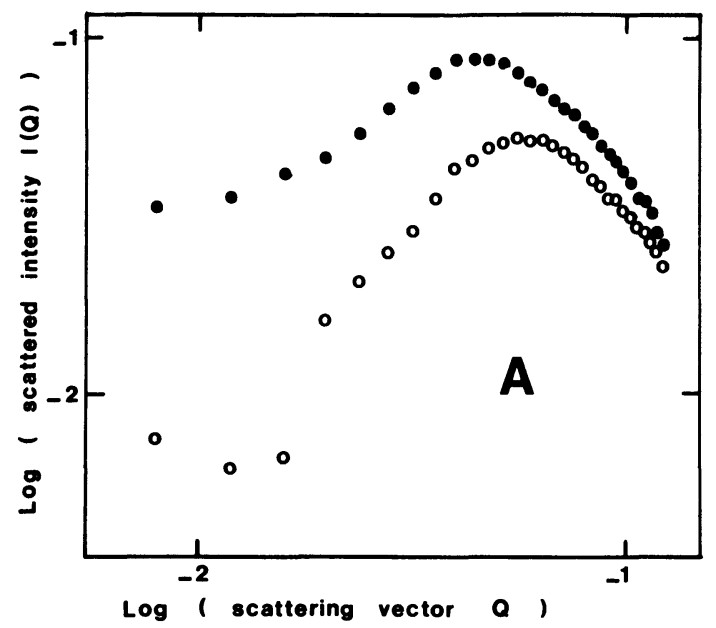

Fig. 8. - Scattering by the micelles of a PEO + SDS solution in region $A$ (circles) compared with the scattering from a pure SDS solution (dots). Note the slight shift of the peak to higher $Q$ (the micelles are $10 \%$ smaller, hence their distances are correspondingly shorter) and the substantial lowering of their osmotic compressibility at low $Q$. PEO concentration $y=0.01 \mathrm{~g} / \mathrm{cm}^{3}$, SDS concentration $\mathrm{x}=0.01 \mathrm{~g} / \mathrm{cm}^{3}$.

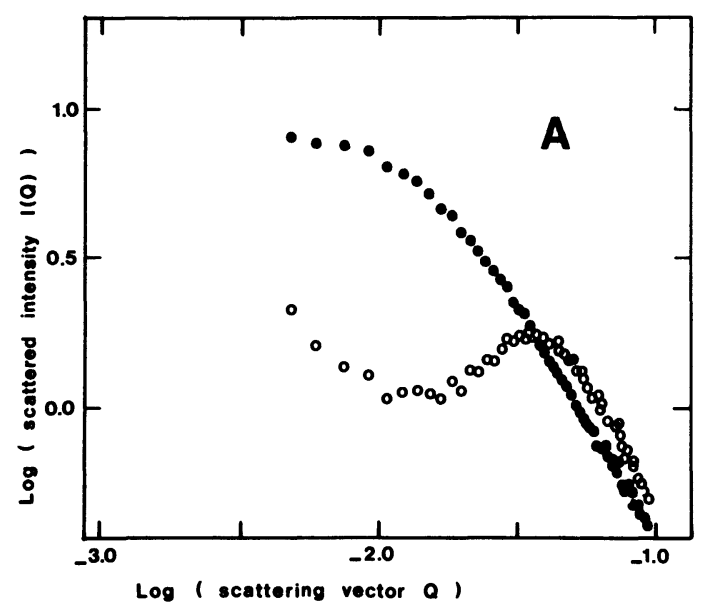

Fig. 9. - Scattering by the macromolecules of a $\mathrm{PEO}+$ SDS solution in region A (circles) compared with the scattering by a pure PEO solution (dots). Note that the main effect of the coupling to the micelles is to depress all the Fourier components with wavevectors between $Q=$ $5 \times 10^{-3}$ and $2 \times 10^{-2} \AA^{-1}$. Yet at lower $Q$ values (osmotic compressibility of the polymer) or at higher ones (local configuration of the chains) the scattered intensity is nearly the same. PEO concentration $y=0.01 \mathrm{~g} / \mathrm{cm}^{3}$, SDS concentration $x=0.01 \mathrm{~g} / \mathrm{cm}^{3}$. 
ations of the concentration of macromolecules are free, i.e. the macromolecules can diffuse through the solution without dragging the micelles with them.

The peak which appears at $Q=3.5 \times 10^{-2} \AA^{-1}$ in the polymer scattering is merely the conjunction of the rise which follows this depression with the normal decay of the polymer scattering at high $Q$. Nevertheless it is noteworthy that its position matches that of the average intermicellar spacings (Fig. 10).

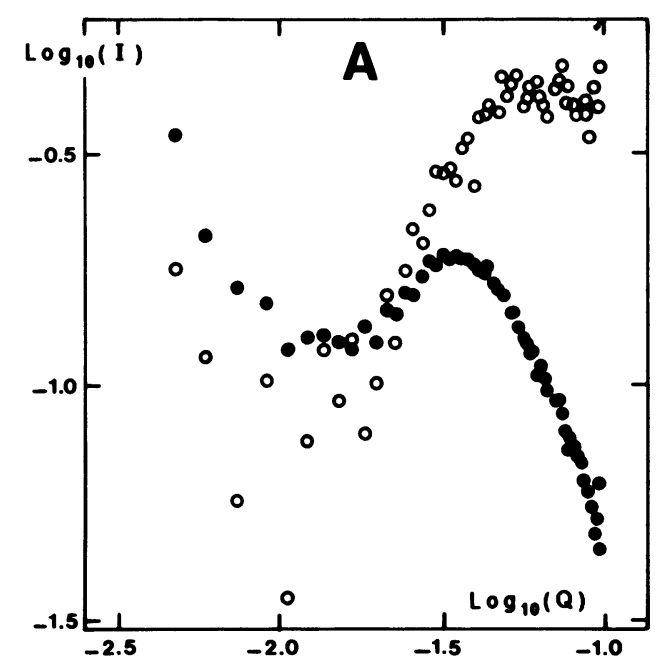

Fig. 10. - The two contrasts for a PEO + SDS solution in region A. Dots : scattering by PEO, the solvent matches the scattering density of deuterated SDS. Circles : scattering by the SDS micelles, the solvent matches the scattering density of PEO. Note that the depression and peak which appear in the PEO curve as a result of the coupling reflect the depression and peak of the SDS curve (intermicellar correlations).

Similar patterns are observed throughout region A; however the effect of the intermicellar correlations on the polymer web fades out at high PEO concentrations (because the concentration of PEO segments tends to become uniform) or at low SDS concentrations (because the intermicellar correlations are lost below $\mathrm{x} \approx 0.005 \mathrm{~g} / \mathrm{cm}^{3}$ ).

A straightforward interpretation of these patterns is that the macromolecules can adsorb on all the micelles without disturbing their electrostatic ordering. This is because, at high PEO/SDS ratios, the free polymer strands which join 2 micelles are long compared to the natural spacings of micelles in the unperturbed array.

3.2 REGION B. - At lower polymer/micelle ratios, the array of micelles is disrupted, and its peak splits into a main peak at lower $Q$ and a subsidiary peak at higher $Q$; in this range the scattering curve of the polymer still gives a peak, which coincides with the main peak of the SDS curve (Fig. 11). A key to understanding the origin of these peaks is provided by their variations with the polymer concentration $y$.

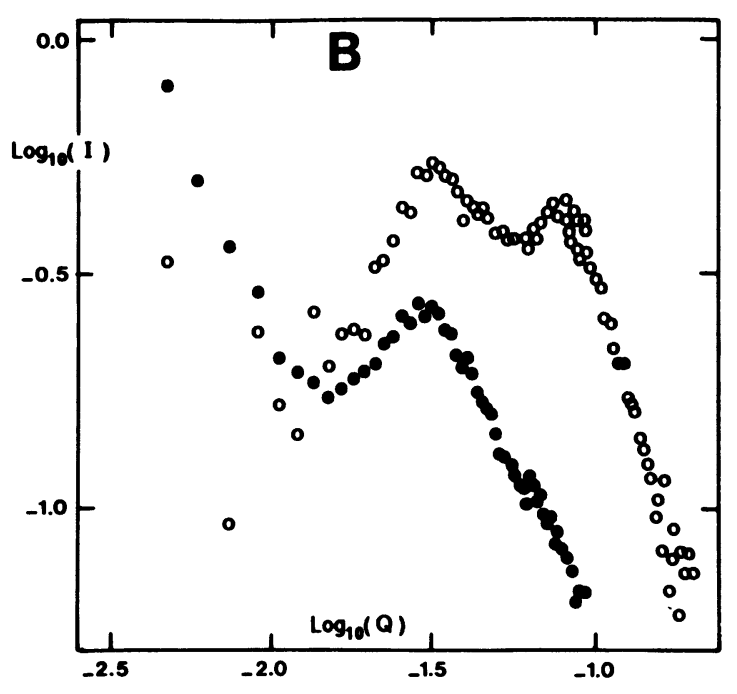

Fig. 11. - Intensities measured in region B : PEO concentration $y=0.005 \mathrm{~g} / \mathrm{cm}^{3}$, SDS concentration $\mathbf{x}=$ $0.01 \mathrm{~g} / \mathrm{cm}^{3}$. Dots : scattering by the PEO macromolecules in the PEO + SDS solution. Circles : scattering by the SDS micelles in the PEO + SDS solution. In this region the macromolecules collect the micelles in necklaces with spacings shorter than those of a free array (shoulder at $Q=0.08 \AA^{-1}$ in the scattering curve of SDS) ; it is argued that the peak at $Q=0.03 \AA^{-1}$ corresponds to distances between necklaces.

As the polymer concentration drops, the 2 peaks for the intermicellar distances keep moving apart, until the lower boundary of region $B$ is reached.

Figure 12 shows the shift of the main peak across region $B$; here the concentration of SDS micelles is kept constant $\left(x=5 \times 10^{-3} \mathrm{~g} / \mathrm{cm}^{3}\right.$, average intermicellar distance for free array $d_{\mathrm{m}}=170 \AA$ ), and the polymer concentration $y$ is varied. We start from the

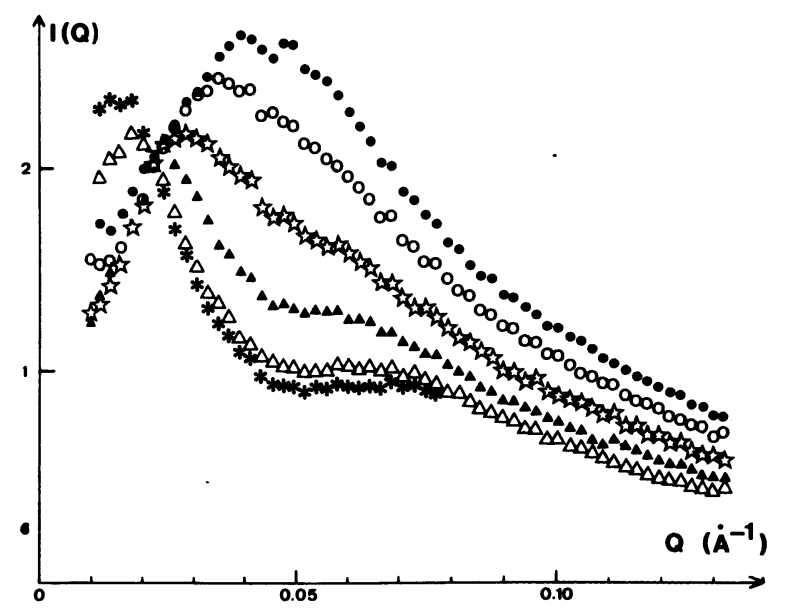

Fig. 12. - Scattering by SDS micelles (concentration $\mathbf{x}=0.005 \mathrm{~g} / \mathrm{cm}^{3}$ ) adsorbed on a PEO web. No contrast matching is used here : both SDS and PEO contribute to the scattering ; this raises the magnitude of the main peak but does not change its position. Dots : PEO concentration $\mathbf{y}=0.01 \mathrm{~g} / \mathrm{cm}^{3}$. Circles : 0.007 . Stars : 0.005 . Filled triangles : 0.003 . Hollow triangles : 0.002 . 
upper boundary, where the mesh size of the web is comparable to $d_{\mathrm{m}}\left(\xi_{\mathrm{b}}=164 \AA\right.$ at $\left.\mathbf{y}=10^{-2} \mathrm{~g} / \mathrm{cm}^{3}\right)$, and cross to the lower boundary where it is much larger $\left(\xi_{b}=800 \AA\right.$ at $\left.y=1.5 \times 10^{-3} \mathrm{~g} / \mathrm{cm}^{3}\right)$. As the polymer is diluted, the main peak shifts to lower $Q$ values (larger distances) according to a $y^{0.55}$ law, while the subsidiary peak shifts more slowly in the opposite direction (higher $Q$ values, shorter distances), approximately as $\mathbf{y}^{-0.1}$ (Fig. 13).

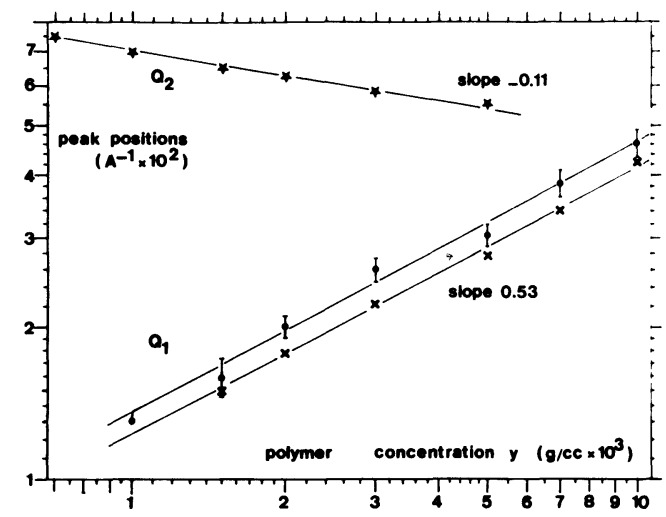

Fig. 13. - Positions of the peaks observed in semidilute $\mathrm{PEO}+$ SDS solutions for various concentrations of PEO (molecular weight $10^{6}$ ). Dots : main peak at lower $Q$, data from D11. Crosses : main peak, data from D17. Stars : subsidiary peak at higher $Q$, data from D17.

The $\mathbf{y}$ dependence of the main peak is close to the square root law found for a similar peak in semidilute solutions of linear polyelectrolytes [42-44]. Therefore the main peak of the SDS scattering curves may be caused by correlations between linear charged objects, i.e. necklaces of SDS micelles. The order of magnitude is right, since the position of this peak is close to the mesh size $\xi_{b}$ of the original web (although the mesh size for a semidilute solution of necklaces may differ somewhat from that of its parent web). Moreover, this interpretation is supported by the fact that a peak is observed at the same position in the PEO scattering curves.

The subsidiary peak at high $Q$ has the same position in both dilute and semidilute solutions (see the following section) ; it corresponds to a distance between consecutive micelles within a necklace. As the number of macromolecules is lowered, these micelles should find themselves pushed closer together in each necklace, and the peak should move to higher $Q$ as $\mathbf{x} / \mathbf{y}$. However this crowding is opposed by the electrostatic repulsions between micelles; instead, the polymer strands stretch to accommodate more micelles per chain without bringing them closer together; hence the weak dependence observed for the position of this peak (Fig. 13).

These 2 sets of distances suggests the following picture : all the micelles are collected on the polymer strands as necklaces, and in this range of compositions the distances between consecutive micelles within a necklace are substantially shorter than those between micelles attached to neighbouring necklaces.

3.3 REgION C. - When the polymer concentration $\mathbf{y}$ is decreased below $\mathbf{x} / 5$, the scattering pattern of SDS returns abruptly to that of a free micellar solution; in this range the scattering curve of PEO follows roughly the $Q^{-1}$ decay of a stretched object, with no evidence of a regular spacing along the chain (Fig. 14).

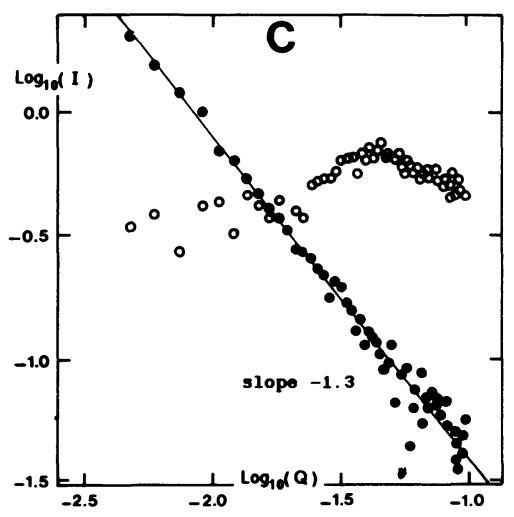

Fig. 14. - Intensities measured in region C : PEO concentration $\mathbf{y}=0.001 \mathrm{~g} / \mathrm{cm}^{3}$, SDS concentration $\mathbf{x}=$ $0.01 \mathrm{~g} / \mathrm{cm}^{3}$. Dots : scattering by the PEO macromolecules. Circles : scattering by the SDS micelles. In this region there are few macromolecules, completely saturated with bound micelles; their scattering curve decays as $Q^{-1.3}$, indicating that they approach the configurations of rigid rods. Most micelles are free, giving a scattering curve which is very close to that of a pure micellar solution (see Fig. 4).

Of course the organization of the micelles in the mixed solution must eventually return to that of a pure surfactant solution when the polymer concentration is low enough; the remarkable feature is that the crossover is abrupt. A similar crossover occurs at the same composition in dilute solutions [3, 4] : there as well, an abrupt saturation of the polymer is observed when $\mathbf{x} / \mathbf{y}$ decreases below a stoichiometric value ; the micelles which cannot be accommodated on the dwindling number of macromolecules are spilled over in the solution where they coexist with the bound micelles.

This saturation occurs when the adsorption of new micelles on the necklaces raises the stretching free energy of the macromolecule and the electrostatic repulsions beyond the gain in adsorption free energy. Then the spacings of micelles in a necklace reach their lower limit, which can be estimated from the position of the subsidiary (intra-necklace) peak observed for SDS at the bottom of region $B$ : we find $85 \AA$ for solutions with no added salt (Fig. 13), and $60 \AA$ at high ionic strength $[3,4]$. 
In region $\mathrm{C}$, the configurations of the saturated necklaces cannot be studied from the scattering curve of SDS, since that is dominated by the excess micelles; however they can be determined from the scattering curve of PEO. This curve shows the same $Q^{-1}$ monotonic decay throughout region $\mathrm{C}$, both at low concentrations of PEO and SDS (Fig. 14) and at concentrations which are higher by one order of magnitude (Fig. 15). The decay is monotonic (instead of the hole and peak observed at low $Q$ in region $B$ ) because the excess micelles screen the internecklace correlations; the $Q^{-1}$ law expresses the stretching of the saturated necklaces by the repulsions between adsorbed micelles. In figure 16 the scattering curve of PEO in streched necklaces is compared to that of the free polymer : the effect is indeed spectacular, as the self avoiding random walk of the free polymer is replaced by a rodlike configuration. Moreover, as the polymer strands stretch, the mesh size becomes smaller; for example, in solutions with $\mathbf{y}=0.002 \mathrm{~g} / \mathrm{cm}^{3}$, it drops from 455 to $191 \AA$ when the solution is loaded with $\mathbf{x}=$ $0.01 \mathrm{~g} / \mathrm{cm}^{3}$ of surfactant.

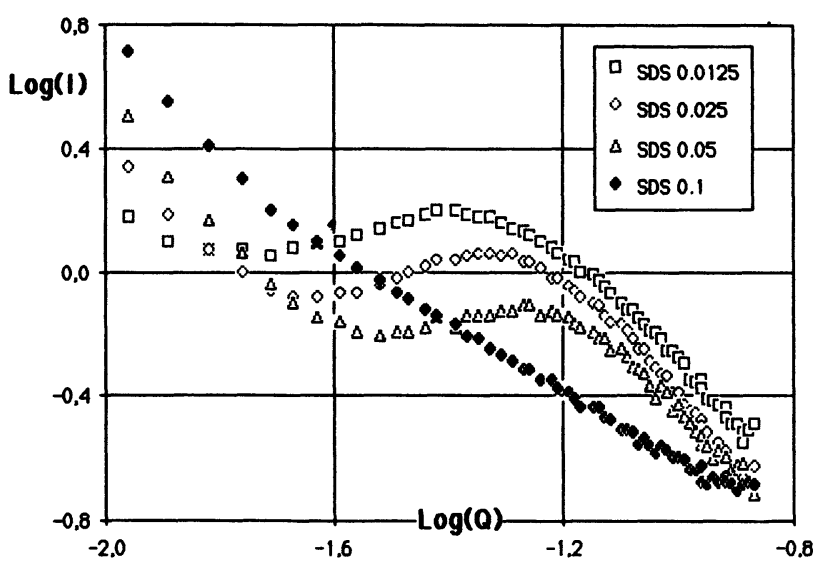

Fig. 15. - Scattering by PEO macromolecules in a crossover from region $A$ to region $C$ : the concentration of PEO macromolecules is fixed at $y=0.01 \mathrm{~g} / \mathrm{cm}^{3}$ and the concentration of SDS micelles is raised from $x=0.0125$ (squares) to 0.025 (lozanges), 0.05 (triangles) and $0.1 \mathrm{~g} / \mathrm{cm}^{3}$ (filled lozanges). The first two concentrations are in region $A$, the next one in region $B$ and the last one in region $\mathrm{C}$.

\section{Interaction diagram.}

In this section the locations of the crossovers are rationalized on the basis of the necklace models for the organization of the solutions. Experimentally, the upper crossover has been found to be a line of slope $0.45 \pm 0.5$ in a Log-Log map of the compositions (Fig. 7) ; it separates two types of structure defined respectively by the interference patterns shown in figures 10 and 11 . The lower crossover is a stoichiometry (slope 1 in Fig. 7) which separates the structures seen in figures 11 and 14 .

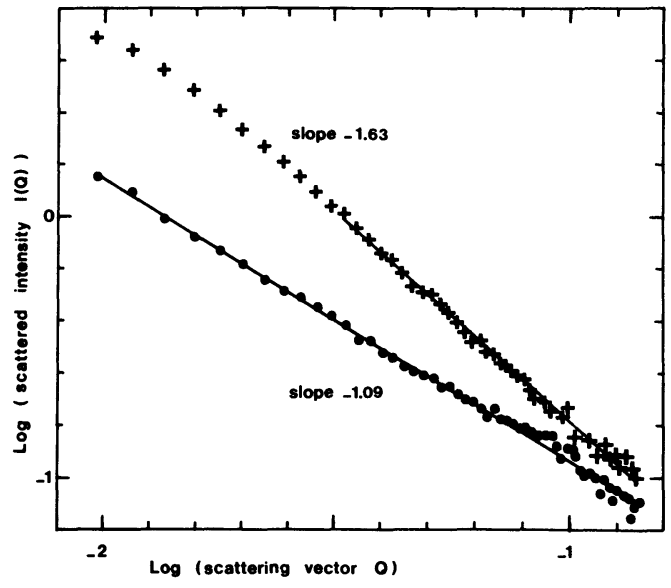

Fig. 16. - Rod-like configuration of saturated necklaces : the scattering curve of PEO macromolecules in a solution located on the saturation line $\left(y=0.002 \mathrm{~g} / \mathrm{cm}^{3}\right.$, $\mathbf{x}=0.01 \mathrm{~g} / \mathrm{cm}^{3}$ ) decays as $Q^{-1.1}$, approximating the $Q^{-1}$ behaviour of rigid rods (dots). By contrast, the $Q^{-1.7}$ decay of the free polymer is typical of a self avoiding random walk (crosses).

4.1 UPPER CROSSOVER. - First consider the region of high polymer concentrations. Here the scattering curves of SDS indicate that the array of micelles is not disorganized by the macromolecules. This should occur when the spacings of micelles in a necklace are longer than the average spacing $d_{\mathrm{m}}=n_{\mathrm{m}}^{-1 / 3}$ of micelles in a free array. A rough estimate for the spacings in a necklace is obtained from the length of a free strand containing $n_{\mathrm{p}} / n_{\mathrm{m}}$ monomers, each of length a ; thus :

$$
d_{\mathrm{m}}=n_{\mathrm{m}}^{-1 / 3} \text { and } d_{\mathrm{p}}=a\left(n_{\mathrm{p}} / n_{\mathrm{m}}\right)^{3 / 5} .
$$

Hence the boundary of this region is obtained for :

$$
d_{\mathrm{p}}=d_{\mathrm{m}} \text { or } \mathbf{y}=C \mathbf{x}^{4 / 9} .
$$

Alternatively, this crossover can be assigned to the mesh size of the original web, which varies as : $\boldsymbol{\xi} \sim \mathbf{y}^{-3 / 4}$. Indeed, when the mesh size of the web is shorter than the average spacing of a free micellar array, it can visit all the points of the array without restricting its configurational entropy ; conversely, when the mesh size becomes substantially larger than the spacings of the array, the adsorption will condense the micelles on the paths of the web. Hence the condition :

$$
\boldsymbol{\xi}_{\mathrm{b}}=d_{\mathrm{m}} \text { and therefore } \mathbf{y}=C^{\prime} \mathbf{x}^{4 / 9} .
$$

Of course these two methods for finding the crossover line are equivalent, because the first one is in effect a method for calculating the mesh size $\xi$ : indeed it looks for points on the web whose distances vary as the $(-1 / 3)$ power of their concentration. They yield the same prefactor $C$; this prefactor agrees with the absolute location of the crossover line (L. Leibler, private communication). 
4.2 STOICHIOMETRY. - Below the upper crossover line, the polymer strands are not long enough to follow the natural spacings of the micellar array ; instead, the macromolecules collect the micelles in necklaces with spacings shorter than those of a free array (shoulder at $Q=0.07 \AA^{-1}$ in Fig. 11). The correlations between such necklaces are similar to those in a semidilute solution of polyelectrolytes [47]; hence the peak at $Q=0.03 \AA^{-1}$ in figure 11 , whose position varies as the mesh size $\xi_{b}$ of the web.

At still lower polymer concentrations, the electrostatic repulsions between micelles prevent them from being adsorbed at intervals shorter than a distance of closest approach $d_{0}$; for PEO + SDS with no added salt, $d_{0}$ is about $100 \AA$ in the necklaces. This distance is reached for a stoichiometric composition of the solutions :

$$
d_{\mathrm{p}}=d_{0} \quad \text { or } \quad \mathbf{y}=\mathbf{x} \cdot\left(d_{0} / a\right)^{5 / 3} .
$$

Below this line, the macromolecules only collect a small fraction of all the micelles ; the remaining, free micelles screen the necklaces from each other.

4.3 OTHER BOUNDARIES. - Besides the two crossovers discussed above, the composition map also shows a vertical and a horizontal boundary; they mark the smallest concentrations below which the PEO + SDS system no longer presents a situation of identical, repelling spheres coupled to an infinite web.

The boundary at $\mathbf{x}=\mathbf{x}_{0}$ is the critical micelle concentration (c.m.c.) of SDS ; it it located at a lower SDS concentration than the c.m.c. of pure SDS because the adsorption of PEO lowers the chemical potential of the micelles [2, 3]. For $\mathbf{x}<\mathbf{x}_{0}$ the solution does not form micelles; for $x \approx x_{0}$ micelles are formed but their concentration is so low that they do not generate appreciable repulsions; they are also smaller than regular SDS micelles [49].

The crossover at $\mathbf{y}=\mathbf{y}^{*}$ is the overlap concentration for PEO macromolecules ; below this concentration macromolecules of molecular weight $M=$ $1.2 \times 10^{6}$ cease to overlap and the solution crosses over to the dilute regime $[18,50]$. This crossover is located at a lower PEO concentration than that for pure PEO because the PEO strands in saturated necklaces are streched, resulting in a smaller mesh size (see the discussion of their scattering curves in region $\mathrm{C}$ ).

\section{Conclusions.}

We have observed the interaction in water of two colloids with very different spatial scales : a polymer web with a mesh size of 200 to $1000 \AA$, and an array of surfactant micelles with radii of $20 \AA$ and separations $\approx 100 \AA$. Through this interaction the struc- tures of both systems are coupled and strongly distorted [51] : in particular, we find one region of compositions where the polymer web adapts itself to the spacings of the micellar array, and another one where the micelles are collected at short intervals along the polymer chains and can no longer keep their average spacings. In all cases, however, the basic step is the formation of linear necklaces of micelles bound to the macromolecules ; these necklaces remain unconnected to each other throughout the range of concentrations explored in this work.

\subsection{COMPARISON WITH LINEAR RESPONSE THEORY.}

- Some of the effects of the polymer-micelles coupling are predicted qualitatively by the linear response theory : for example the peak observed in the scattering curve of the polymer at the position of intermicellar spacings (Fig. 9) can indeed be obtained in this model if the coupling is strong and the SDS concentration large enough (Fig. 5). However the differences are severe. Indeed the observed coupling produces mostly depressions of the scattering curves (Figs. 8 and 9) rather than the increases predicted by the theory (Figs. 5 and 6). Moreover the condensation of the micelles of the polymer in region $B$, with separate peaks for the correlations within a necklace and between necklaces (Fig. 11), is just not within the frame of linear response. Finally, the existence of 3 regimes with sharp crossovers between them, and the asymmetry of the observed couplings (one component follows the spatial arrangement of the other) indicate that the linear theory is basically inappropriate.

What is wrong in this analysis is the assumption that each micelle interacts weakly with all polymer strands in its vicinity, and vice versa. Indeed, the interaction occurs through the adsorption of polymer strands on the micellar surfaces, and it is well known that polymer adsorption can be a highly non linear process. The adsorption of polymers on small spheres is particularly odd, because a macromolecule which meets a sphere will always lower its free energy by saturating the sphere's surface $[45,46]$; it is unlikely that 2 macromolecules or 2 remote sections of the same chain will be allowed in the vicinity of the same sphere, unless the overall density of macromolecules is so high that $\xi_{b}$ is smaller than the sphere's radius [48].

Consequently the system does not form the « gel » predicted by linear response theory. Instead, the configurational entropy of the macromolecules leads to the formation of single chain necklaces. Still, one could consider another route to gelation, where saturated necklaces would be connected to each other through excess spheres [45]. This does not occurs in PEO + SDS systems, presumably because the repulsions between micelles would offset the corresponding gain in adsorption free energy. 
5.2 COMPARISON WITH GELATION AND FLOCCULATION. - In colloid science there are many situations which involve long macromolecules and small particles; of particular relevance here is the process where dissolved macromolecules are used to collect particles dispersed in water (flocculation). The usual situation is that where the macromolecules are attracted to the surfaces of the particles; then it is presumed that each macromolecule adsorbs on more than one particle, creating bridges between them ; the growh of a piece of gel (the « floc») will then occur if there is more than one bridge per sphere.

This picture of a floc as a piece of gel held together by macromolecular bridges is obviously similar to the necklace structures found here for polymers adsorbed on micelles; however it also reveals a stricking difference, as the necklaces of PEO + SDS solutions never turn into a gel. This remarkable difference in behaviour can be tracked down to the topology of the binding : why is it that a particle in a floc can bind many macromolecules, whereas a SDS micelle can bind only one? The answer to this question may set some of the limits to common flocculation processes.

Obviously, the necklace structures observed for PEO + SDS solutions represent the equilibrium situ- ation for very large macromolecules adsorbed on small spheres. Hence the formation and separation of a gel would require either non equilibrium conditions or a reversal in the hierarchy of spatial scales, i.e. small macromolecules on large spheres. Then the mechanism which would favor gel formation appears clearly : in the initial phase of mixing, with hydrodynamic or composition gradients, a sphere which has started binding a macromolecule may meet another one before its surface has been saturated by the first one ; this will occur more often if the spheres are large. This mechanism where a sphere binds all macromolecules in its vicinity is close to that postulated in the linear response theory. In this respect, it is remarkable that the non equilibrium structures (gels, flocs) are produced according to linear processes, while the equilibrium ones are dictated by non linear effects.

\section{Acknowledgments.}

We have enjoyed a number of discussions (mostly controversial) with J. F. Joanny, L. Leibler, P. A. Pincus, S. Alexander and T. Witten. It is also a pleasure for us to acknowledge the help and support from ILL and LLB staff, including J.P. Cotton, J. Teixeira and P. Timmins.

\section{References}

[1] Rовв, I. D., in Anionic surface : physical chemistry of surfactant action, Lucassen Reynders, E. J. editor (Dekker) 1981.

[2] Cabane, B., J. Phys. Chem. 81 (1977) 1639.

[3] Cabane, B., Duplessix, R., J. Physique 43 (1982) 1529.

[4] Cabane, B., Duplessix, R., Colloids Surfaces 13 (1985) 19.

[5] Reiss Husson, F., Luzzati, V., J. Phys. Chem. 68 (1964) 3504.

[6] Reiss Husson, F., Luzzati, V., J. Colloid Interface Sci. 21 (1966) 534.

[7] Wennerström, H., Lindman, B., Phys. Rep. 52 (1979) 1.

[8] Hayter, J. B., Penfold, J., J. Chem. Soc. Faraday Trans. I 77 (1981) 1851.

[9] Hayter, J. B., Penfold, J., Colloid Polym. Sci. 261 (1983) 1022.

[10] Cabane, B., Duplessix, R., Zemb, T., J. Phys. 46 (1985) 2161.

[11] Mukerjee, P., MÝsels, K. J., Critical micelle concentrations 01 aqueous surfactant systems (Nat. Stand. Ref. Data System 1971) Superintendant of documents, US Government Printing office, Washington, D.C. 20402, USA.

[12] Goddard, E. D., Phillips, T. S., Hannan, R. B., J. Soc. Cosmet. Chem. 26 (1975) 461.

[13] Shirahama, K., Ide, N., J. Colloid Interface Sci. 54 (1976) 450.
[14] Guinier, A., Fournet, G., Small angle scattering of $X$ rays (Wiley, New York) 1955.

[15] Hansen, J. P., MCDonald, I. R., Theory of Simple Liquids (Academic press, London) 1976.

[16] Hayter, J. B., Penfold, J. Mol. Phys. 42 (1981) 109.

[17] Hansen, J. P., Hayter, J. B., Mol. Phys. 46 (1982) 651.

[18] DE GENNES, P. G., Scaling concepts in polymer physics (Cornell University Press, Ithaca) 1979.

[19] Edwards, S. F., Proc. Phys. Soc. 88 (1966) 265.

[20] Broseta, D., Leibler, L., LAPP, A., Strazielle, C., to be published.

[21] Joanny, J. F., Polymer 21 (1980) 71.

[22] Dreger, E. E., Keim, G. I., Miles, G. D., ShedLOVSKY, L., Ross, J., Ind. Eng. Chem. 36 (1944) 610.

[23] Miles, G. D., Shedlovsky, L., J. Phys. Chem. 48 (1944) 57.

[24] Vijayendran, B. R., J. Colloid Interface Sci. 60 (1977) 418.

[25] Rosen, M. J., J. Colloid Interface Sci. 79 (1981) 587.

[26] Hendrikx, Y., Charvolin, J., J. Physique 42 (1981) 1427.

[27] Strazielle, C., Makromol. Chem. 119 (1968) 50.

[28] Layec, Y., Layec-Raphalen, M. N., J. Physique Lett. 44 (1983) L-121.

[29] GÜner, A., GÜVEn, O., Makromol. Chem. 179 (1978) 2789. 
[30] Strazielle, C., private communication.

[31] Jacrot, B., Rep. Progr. Phys., 39 (1976) 911.

[32] Cabane, B., in Colloides et Interfaces, Veyssié, M. and Cazabat, A. M. editors (Editions de Physique, France) 1984.

[33] LOVESEY, S. W., Theory of neutron scattering from condensed matter (vol. 1) (Clarendon Press, Oxford) 1984.

[34] Corkill, J. M., Goodman, J. F., Walker, T., Trans. Faraday Soc. 63 (1967) 768.

[35] Musbally, G. M., Perron, G., Desnoyers, J. E., J. Colloid Interface Sci. 48 (1974) 494.

[36] Caron, G., Perron, G., Lindheimer, M., DesNOYERS, J. E., J. Colloid Interface Sci. 106 (1985) 324.

[37] Vikingstad, E., Skauge, A., Hфiland, A., J. Colloid Interface Sci. 66 (1978) 240.

[38] Benjamin, L., J. Phys. Chem. 70 (1966) 3790.

[39] Mukerjee, P., J. Phys. Chem. 66 (1962) 1733.

[40] Lepori, L., Mollica, V., J. Polym. Sci. Polym. Phys. Ed. 16 (1978) 1123.

[41] Ragnetti, M., Geiser, D., Höcker, H., OberTHÜR, R. C., Makromol. Chem. 186 (1985) 1701.

[42] Niehrlich, M., Williams, C. E., Boué, F., CotTON, J. P., DAOUd, M., Farnoux, B., Jan-
NinK, G., Picot, C., MoAn, M., WolfF, C., Rinaudo, M., De Gennes, P. G., J. Physique 40 (1979) 701.

[43] Drifford, M., Dalbiez, J. P., J. Chem. Phys. 88 (1984) 5368.

[44] Koyama, R., Macromolecules 19 (1986) 178.

[45] Alexander, S., J. Physique 38 (1977) 977.

[46] Pincus, P. A., Sandroff, C. J., Witten, T. A. Jr., J. Physique Lett. 45 (1984) 725.

[47] HAYTER, J., JANNINK, G., BROCHARD-WyART, F., De Gennes, P. G., J. Physique Lett. 41 (1980) L-451.

[48] See also the arguments developed by Witten for the gelation of ionomers: WITTEN, T. A. Jr., COHEN, M. H., Macromolecules 18 (1985) 1915.

[49] ZANA, R., LANG, J., LiAnOs, P., in Microdomains in polymer solutions, Dubin, P. editor (Plenum) 1985.

[50] Wells, J. D., J. Chem. Soc. Faraday Trans. I 80 (1984) 1233.

[51] For another example of coupling between two colloids of different scales, see: YoshIMURA, S., HaChisu, S., J. Physique Colloq. 46 (1985) C3115. 